\title{
Loss of Control Prevention and Recovery: Onboard Guidance, Control, and Systems Technologies
}

\author{
Christine M. Belcastro ${ }^{1}$ \\ NASA Langley Research Center, Hampton, Virginia, 23681
}

\begin{abstract}
Loss of control (LOC) is one of the largest contributors to fatal aircraft accidents worldwide. LOC accidents are complex in that they can result from numerous causal and contributing factors acting alone or (more often) in combination. These LOC hazards include vehicle impairment conditions, external disturbances; vehicle upset conditions, and inappropriate crew actions or responses. Hence, there is no single intervention strategy to prevent these accidents. NASA previously defined a comprehensive research and technology development approach for reducing LOC accidents and an associated integrated system concept. Onboard technologies for improved situation awareness, guidance, and control for LOC prevention and recovery are needed as part of this approach. Such systems should include: LOC hazards effects detection and mitigation; upset detection, prevention and recovery; and mitigation of combined hazards. NASA is conducting research in each of these areas. This paper provides an overview of this research, including the near-term LOC focus and associated analysis, as well as preliminary flight system architecture.
\end{abstract}

\section{Introduction}

L OSS of control (LOC) is one of the largest contributors to fatal aircraft accidents worldwide. As shown in Figure 1, in-flight LOC was the largest fatal accident category for jet transport accidents worldwide from 2001 through 2010, and resulted in 20 accidents and 1,841 fatalities. ${ }^{1}$ LOC is a significant contributor to accidents and fatalities across all vehicle classes, operational categories, and phases of flight. ${ }^{2,3}$ It is also a complex event, usually resulting from multiple causal and contributing factors that can occur individually or (more often) in combination. There is therefore no single intervention strategy that can be readily identified to prevent LOC accidents.

A detailed analysis of aircraft accidents was performed to identify worst case combinations of LOC precursors and how they sequence in time. ${ }^{4}$ The analysis performed in Ref. 4 included accidents that involved vehicle upsets, as well as those involving failures, impairment, or damage to the flight control capability of the aircraft or to the vehicle airframe (when the damage was sufficient to alter vehicle dynamics and control characteristics) whether or not these factors led to an upset. The data set used in the analysis consisted of 126 accidents that resulted in 6087 fatalities during the period from 1979-2009. The analysis included the identification of worst case combinations of causal and contributing factors and a detailed compilation of LOC sequences based on temporal ordering of causal and contributing factors. A list of the top 10 LOC summarized sequences was developed, which represents $86.5 \%$ of the accidents considered in the paper. A set of 7 generalized LOC sequences was also defined, which are representative of $88.9 \%$ of the accidents considered in the study. A preliminary identification of potential future LOC risks was also presented.

Based on the above LOC problem analysis, a comprehensive research and technology development approach ${ }^{5,6}$ for reducing LOC accidents was developed to provide prevention, avoidance, detection, mitigation, and recovery capabilities across a wide spectrum of LOC precursor combinations and sequences. The approach includes the development of (i) modeling and simulation technologies for characterizing vehicle dynamics and control characteristics under off-nominal precursor conditions associated with LOC events; (ii) vehicle health management (VHM) technologies for the detection, identification, characterization, and containment of airframe and system failures and damage (as well as their prevention though improved maintenance, inspection, and vehicle design);

\footnotetext{
${ }^{1}$ Senior Research Engineer, Dynamic Systems and Control, 8 Langley Blvd., MS 308, and AIAA Associate Fellow.
} 
(iii) flight safety assessment and resilient guidance and control technologies for the rapid assessment of off-nominal condition effects and their mitigation; and (iv) crew interface technologies for improved situation awareness (SA) and variable autonomy under off-nominal conditions. An associated high-level integrated system concept, called the Aircraft Integrated Resilient Safety Assurance and Failsafe Enhancement (AIRSAFE) System, was defined as part of the approach. Figure 2 depicts the AIRSAFE System concept.



Figure 1. Aircraft accident statistics for worldwide commercial jet fleet, 2001 - 2010.

The core subsystems include vehicle health management (shown in green), vehicle flight safety management and resilient guidance and control (shown in blue), and crew-system interfaces (shown in yellow). Onboard modeling capability is reflected by purple. Multi-colored boxes represent shared functions between the associated subsystems. A more detailed description of the functions within each block of Fig. 2 was also provided in Refs. 5 and 6.

This paper provides an overview of the research being conducted by the Vehicle Systems Safety Technologies (VSST) Project within the National Aeronautics and Space Administration's (NASA) Aviation Safety Program (AvSP), with an emphasis on research that addresses LOC. Section II provides a general overview of the VSST Project. Section III provides an overview of VSST research that addresses LOC, and a detailed overview of guidance, control, and systems (GCS) technologies being developed for LOC prevention and recovery. The LOC hazards being addressed in the near-term are presented with the associated analysis, and a preliminary GCS architecture for technology integration and implementation is presented. Section IV illustrates the potential effectiveness of these technologies and briefly discusses their evaluation. Section V completes the paper with a summary and some concluding remarks. 


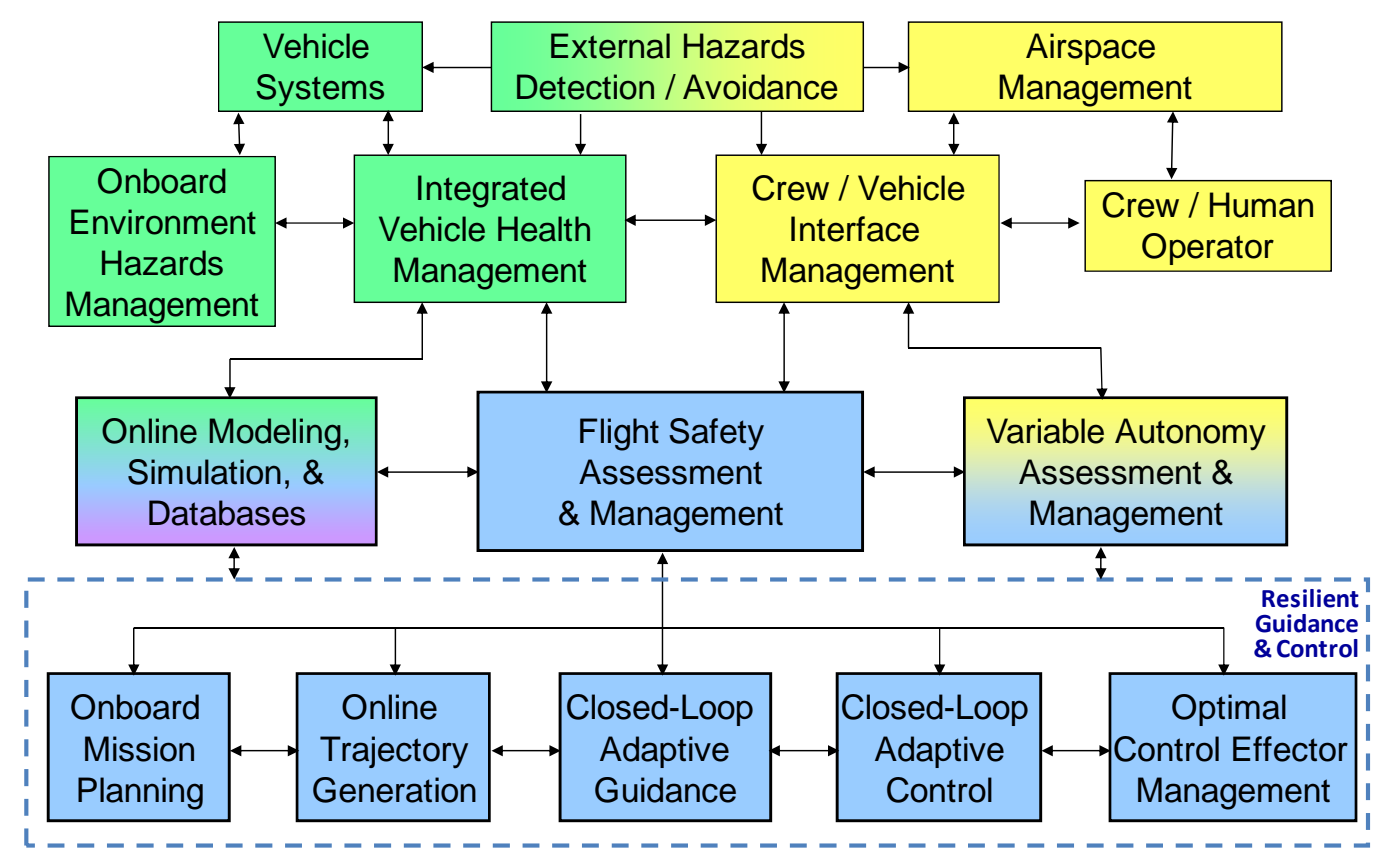

Figure 2. Aircraft Integrated Resilient Safety Assurance and Failsafe Enhancement (AIRSAFE) System.

\section{Vehicle Systems Safety Technologies (VSST) Project Overview}

The VSST Project is developing technologies for improved vehicle and vehicle systems safety under current and future safety risks. The VSST Project is focused on three primary safety risk areas, as illustrated in Fig. 3.

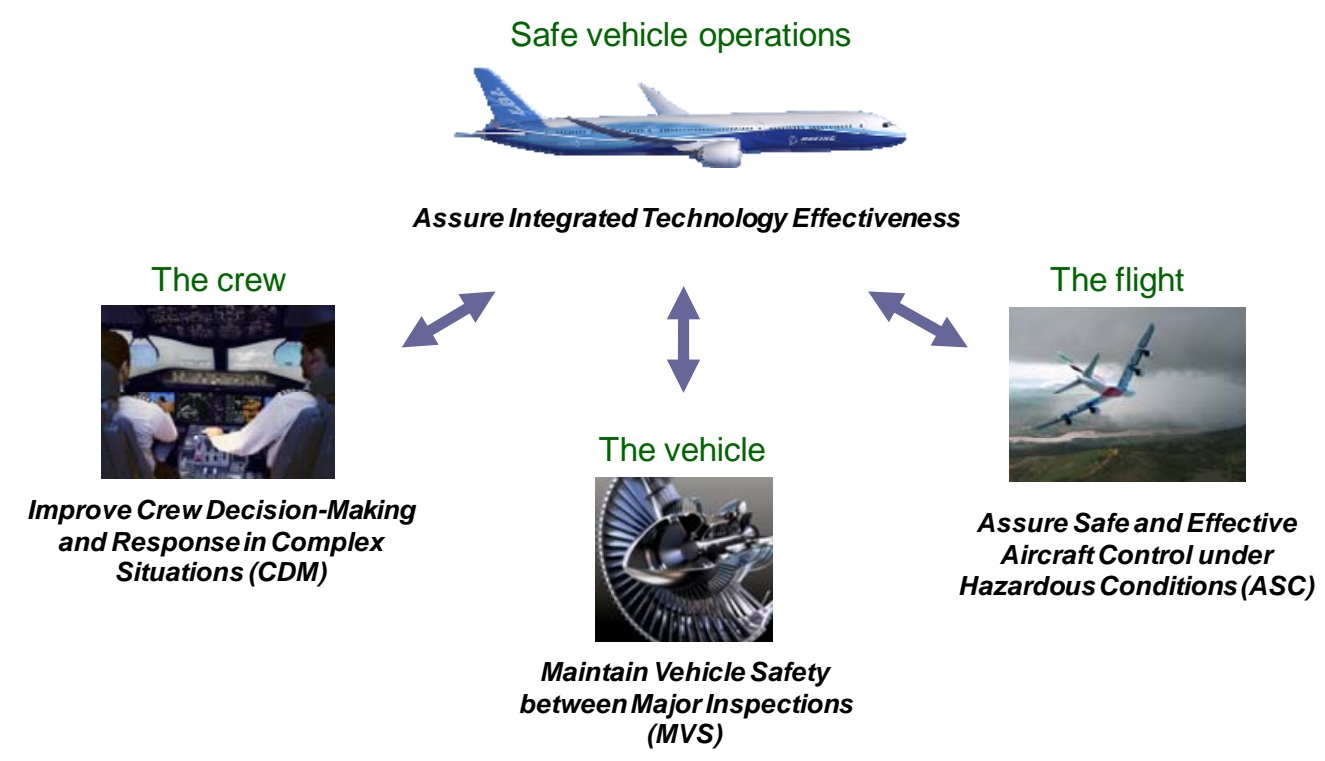

Reduce current risks; Identify and proactively mitigate new risks

Figure 3. Vehicle Systems Safety Technologies (VSST) Project overview. 
Vehicle-centric safety risk focuses on the crew, the vehicle, and the flight. The flight crew and aircraft comprise the first and second causal factor of commercial transport accidents worldwide, and LOC is the leading pre-crash consequence associated with these and other causal and contributing factors. ${ }^{7}$ These three risk areas therefore comprise the three technical challenges being addressed by the VSST Project: 1.) Improve crew decision-making under complex situations (CDM); 2.) Maintain vehicle safety between major inspections (MVS); and 3.) Assure safe and effective control under hazardous conditions (ASC).

CDM research focuses on the development of advanced flight deck technologies to improve SA, ensure pilot engagement on safety-critical tasks, and provide integrated information management and critical decision support. The development of pilot proficiency standards for maintaining manual handling skills under increasing flight deck automation is also a subject of research within CDM. Complex flight situations related to unexpected events and operations under the Next Generation Air Transportation System (NextGen) ${ }^{8-9}$ is a key aspect of the research within CDM.

MVS research focuses on the development of sensors and diagnostic tools for preventing critical vehicle and system failures, high-temperature engine sensor systems for reliable engine health monitoring, and airframe and engine materials and coatings that detect and minimize damage related to fatigue, fracture, delamination, and corrosion.

ASC research focuses on the development of vehicle dynamics modeling and simulation technologies for characterizing LOC precursor conditions, and onboard GCS technologies for LOC prevention and recovery. Vehicle dynamics modeling and simulation methods are being developed for characterizing vehicle upset and impairment conditions. Existing external disturbance models will also be integrated into the resulting simulations. The GCS technologies are being developed to mitigate hazardous conditions that can lead to LOC. These technologies will be described in more detail in Section III.

Assuring safe vehicle operations at the top of Fig. 3 represents a parallel effort on comprehensively evaluating and validating the technologies developed within CDM, MVS, and ASC to assure that these technologies are effective in improving safety. Validation of VSST technologies developed for LOC prevention and recovery is the subject of a companion paper (see Ref. 30). This will also be briefly discussed in Section IV.

\section{VSST Research and Technology Development for LOC Prevention and Recovery}

Due to LOC complexity, significant reduction of LOC accidents will require a multi-pronged coordinated effort that includes improved crew training and onboard systems technologies. This section provides an overview of the research approach being taken by NASA within the VSST Project for LOC prevention and recovery, with an emphasis on the GCS technologies development. Technology needs are discussed first, followed by the near-term research focus, an overview of VSST technologies that are being developed to address LOC, and a detailed description of GCS technologies that are under development for LOC prevention and recovery.

LOC can be described as motion that is: outside the normal operating flight envelopes; not predictably altered by routine pilot control inputs; characterized by nonlinear effects, such as kinematic/inertial coupling; disproportionately large responses to small state variable changes, or oscillatory/divergent behavior; likely to result in high angular rates and displacements; and characterized by the inability to maintain heading, altitude, and wingslevel flight. ${ }^{10}$ LOC is therefore fundamentally a dynamics and control problem, but there are many causal and contributing factors that can lead to LOC. The primary causes include: entry into a vehicle upset condition; reduction or loss of control power; changes to the vehicle dynamic response in relation to handling/flying qualities; and combinations of these causes. There are numerous factors that have historically led or contributed to LOC. These can be grouped into three major categories: adverse onboard conditions, external hazards and disturbances, and abnormal flight conditions (or vehicle upsets). LOC causal and contributing factors within these categories are summarized in Fig. 4. Adverse onboard conditions includes vehicle problems (i.e., impairment, failures, or damage) and inappropriate crew response. External hazards and disturbances consist of inclement weather conditions, atmospheric disturbances, and obstacles that require abrupt maneuvering for avoidance. Vehicle upset conditions include a variety of off-nominal or extreme flight conditions and abnormal trajectories. The complexity of LOC is clearly illustrated in Fig. 4, particularly considering that many LOC accidents involve combinations of the causal and contributing factors that are listed. 


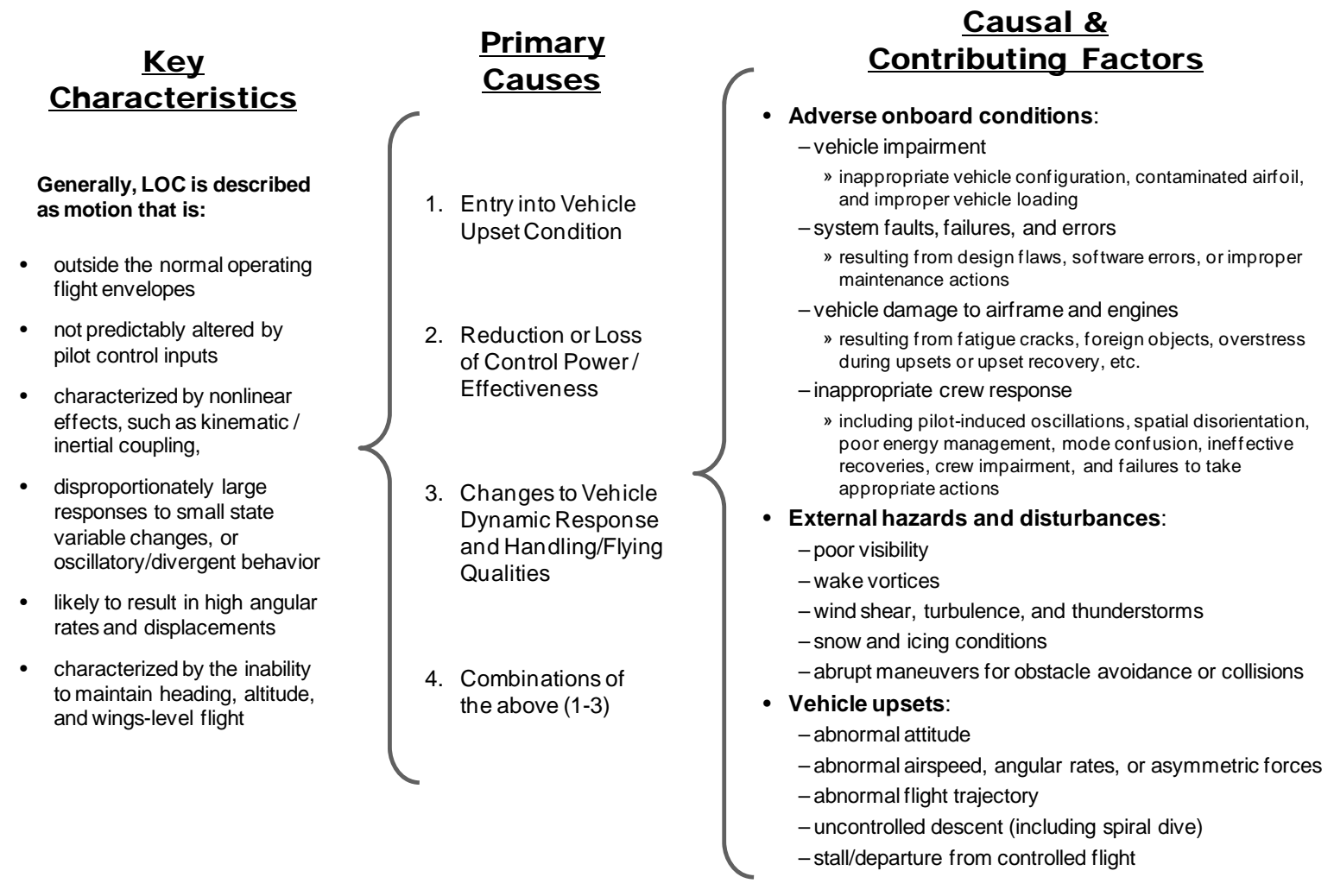

Figure 4. LOC key characteristics, primary causes, and causal \& contributing factors.

\section{B. Technology Needs for LOC Prevention}

Inappropriate crew response is often listed as a causal or contributing factor in LOC accidents. Two primary limitations can be attributed to this problem: limitations in crew training related to LOC, and limitations of onboard systems in providing SA to the crew under LOC precursor conditions. Current crew training under LOC conditions is limited because of model limitations for full stall and other upset conditions, failures and damage, and environmental hazards. There is also evidence that manual handling skills under increasing automation in the flight deck are not being reinforced through crew training. ${ }^{11}$ Current onboard systems do not clearly inform of impending loss of control or provide effective control under hazardous conditions.

Current aircraft guidance and control systems are designed independently for operation under nominal flight conditions, and can disengage unexpectedly under off-nominal conditions. Disengagement of these systems can occur without advance warning, during periods of peak workload or confusion by the crew, and often result in control input transients that further exacerbate conditions that can lead to LOC. Current envelope protection systems provide limited capabilities in that they are designed for nominal aircraft based on a priori fixed limits and depend upon valid sensor inputs. In particular, they are generally not effective under vehicle impairment conditions, and may disengage under extreme upset conditions and control surface or sensor failures. ${ }^{12}$

Future potential LOC risks must also be considered in identifying technology needs. Increasing demands on the national airspace has necessitated development of and transition to the Next Generation Air Transportation System (NextGen). Operation under NextGen will include high-density operations, efficient trajectories for fourdimensional operations, and self-spacing. These operational changes could require a higher demand on the flight crew, and could result in a higher number of external hazards encounters (e.g., wake vortices), particularly in the terminal area. Future aircraft are being developed for high efficiency and reduced weight. Future airframes are therefore likely to be more flexible, with a higher susceptibility to aeroelastic and aeroservoelastic structural modes. Composite materials are being increasingly incorporated into new vehicle designs, and future aircraft configurations could depart from the conventional tube-and-wing design. Damage and damage propagation properties associated with new materials could be very different from those associated with current materials, and vehicle upset properties 
associated with new vehicle configurations could also be very different from those associated with conventional aircraft designs. All of these factors provide a potential for increased LOC risk unless proactive steps are taken to define, prioritize, and mitigate the associated hazards.

Improved modeling and simulation technologies are needed to support improved crew training under LOC conditions, as well as for the development and evaluation of onboard systems technologies. Enhanced simulations must be capable of characterizing representative LOC scenarios, and these simulations must be representative of current and future vehicle classes. Improved training methods are needed to ensure that manual handling skills are retained despite increasing levels of flight deck automation.

Onboard systems technologies for improved SA, guidance, and control under operationally relevant LOC scenarios are needed for LOC prevention and recovery. Such systems should include: external hazards effects detection and mitigation; upset detection, prevention, and recovery; vehicle impairment detection and mitigation, and an integrated approach that enables the detection and mitigation of multiple hazards simultaneously.

\section{Near-Term LOC Research Focus}

The complexity of LOC as illustrated in Fig. 4 requires that key causal and contributing factors be identified as a focus for near-term VSST research. This near-term focus is based on the analysis presented in Ref. 4, as well as a recent analysis of the Commercial Aviation Safety Team (CAST) Joint Safety Analysis Team (JSAT) for Loss of Aircraft State Awareness. Although a final report on this analysis is not yet available, a related report on mode awareness and energy state management within flight deck automation is available. ${ }^{13}$ In order to remove the potential for including precursor effects that may have already been resolved or masking emerging precursor combinations, only accident data over the most recent 10 -year period was assessed from Ref. 4. This near-term analysis was based on 64 accidents (with 2821 fatalities) that occurred over the time period 2000-2009. Figure 5 shows a scatter plot of this analysis as well as a selected potential future risk from NextGen, and illustrates the nearterm LOC problem focus. As indicated in the figure, near-term VSST research will focus on multiple hazards stemming from vehicle problems, external hazards, and crew errors. Vehicle-related hazards include system faults and failures and vehicle impairment conditions (airframe and engine) resulting from icing effects. External hazards include icing conditions and wake vortices, with wakes being considered as a potential future hazard resulting from high-density terminal area operations. Crew-related hazards include loss of energy state awareness (LESA) and spatial disorientation (SD), both of which can lead to various upsets including stalls and departures.

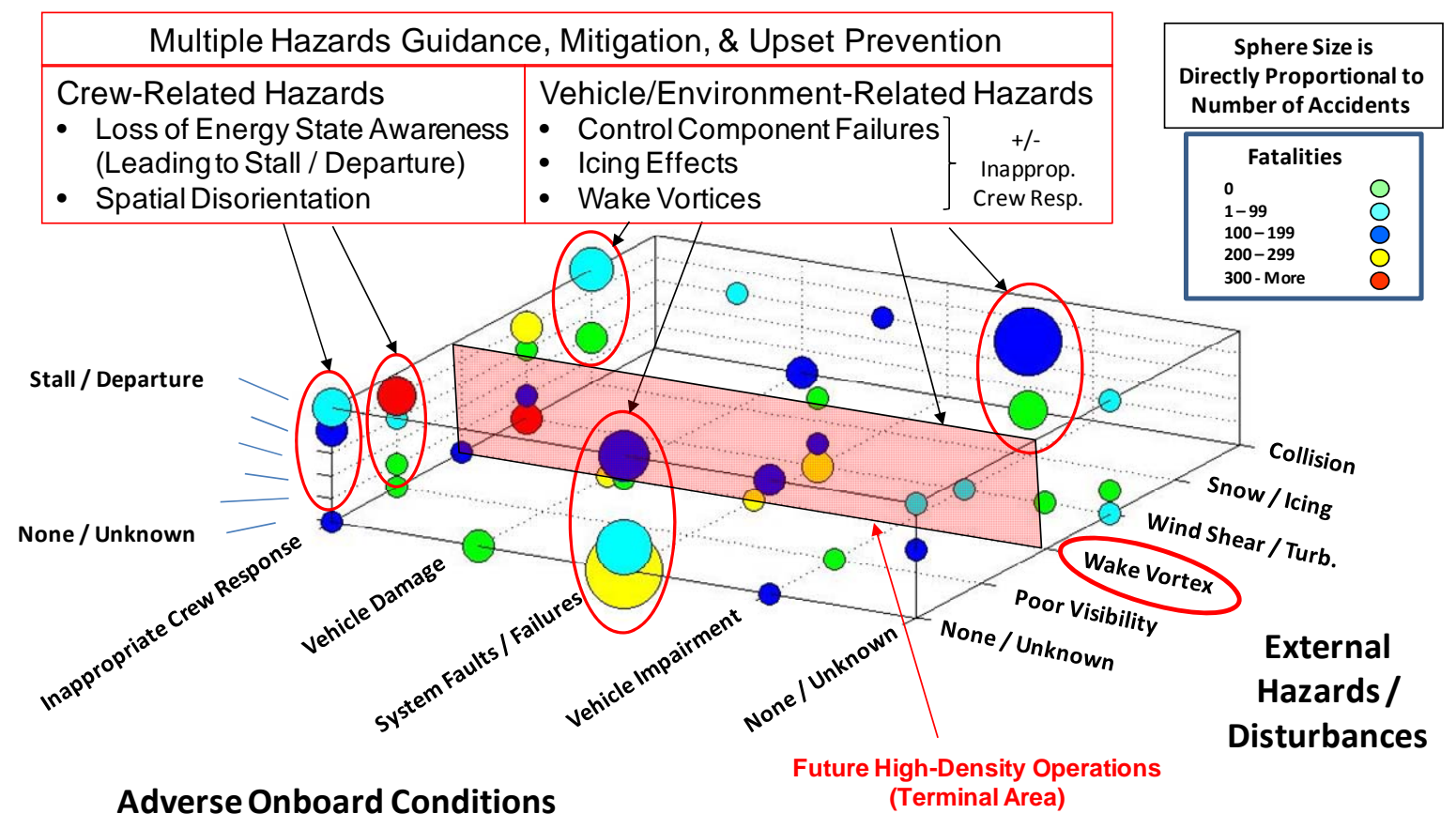

Figure 5. Near-term LOC problem focus. 


\section{VSST LOC Research Approach}

A comprehensive research and technology development approach for reducing LOC accidents was defined in Ref. 5 to provide prevention, avoidance, detection, mitigation, and recovery capabilities across a wide spectrum of LOC precursor combinations and sequences. This approach provides multiple opportunities of breaking LOC sequences at nearly every stage, which maximizes the potential to prevent LOC accidents. Figure 6 depicts the basic idea of this approach, as illustrated for an example generalized LOC sequence identified in Ref. 4. This generalized sequence is representative of 20 accidents $(16 \%)$ and 907 fatalities (15\%) from the analysis of Ref. 4. The LOC sequence of Figure 6 is initiated by either a vehicle impairment/damage or system fault/failure condition or an external hazard or disturbance, such as wind shear or icing (the latter of which can result in vehicle impairment). The second element in this sequence is an inappropriate crew response (including an inappropriate action, control input, or inaction). The inappropriate response could result from poor SA under the vehicle impairment or external hazard condition, spatial disorientation under poor visibility conditions, mode confusion associated with flight deck automation, or some other condition (e.g., crew incapacitation). The third element of this sequence is a vehicle upset. As indicated in Fig. 6, the LOC sequence can be broken if effective intervention strategies can be developed to avoid/detect adverse vehicle and external hazard conditions, if vehicle problems and external hazards can be mitigated when they occur, and if upset recovery can be accomplished when prevention is not successful.

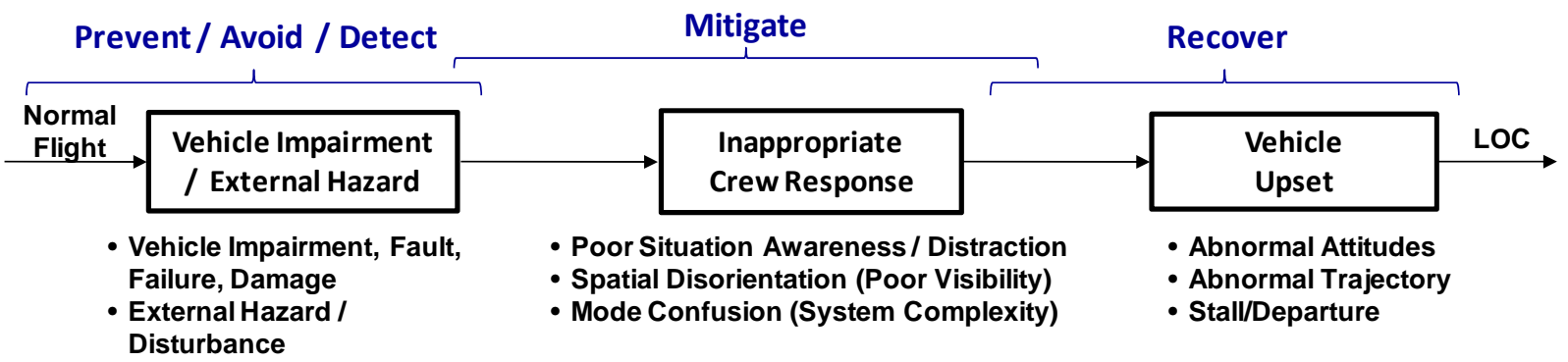

Figure 6. Comprehensive approach to breaking LOC sequences (Ref. 5).

Figure 7 provides a summary of the VSST technologies under development that relate to LOC prevention and recovery in the context of the near-term focus of Fig. 5 and the research approach of Fig. 6. VSST LOC-related research focuses on the prevention and/or mitigation of inappropriate crew response, vehicle impairment conditions, and atmospheric disturbances.

Inappropriate crew response is often a causal or contributing factor in LOC accidents. Prevention of inappropriate crew actions is being addressed through improved training, situation awareness, and flight deck countermeasures. Improved training is enabled through enhanced vehicle dynamics models and simulations that more accurately characterize LOC precursor effects and by establishing training standards for retaining improved manual flying proficiency. Improved situation awareness is enabled by ensuring that information is provided on the current aircraft state, including energy and attitude states as well as any vehicle impairment conditions and the associated dynamics and control implications. Countermeasures for preventing and mitigating the effects of spatial disorientation and crew distraction are also being developed.

Vehicle impairment resulting from system and component failures or icing effects can also contribute to LOC. Methods for failure prevention are being developed under MVS and are accomplished through improved design and early detection of anomalies. Real-time detection and mitigation of failures that do still occur, particularly those that directly impact vehicle dynamics and control characteristics, are being developed under ASC. Icing effects detection, identification, and mitigation are also under development within ASC. While the detection of environmental hazards is not explicitly being addressed under VSST, methods for the mitigation of their effects is being considered under ASC, with a focus on wake vortex and turbulence encounters. Turbulence is included because it often accompanies icing conditions. Multiple hazards effects from all of these categories are also being addressed under ASC, as well as methods for assessing their implications on flight safety. Specific aspects of flight safety being addressed include identification of maneuverability constraints resulting from vehicle impairment conditions, detection of the onset of a vehicle upset condition (in the presence of other hazards, including 
inappropriate control inputs by the crew), and LOC prediction methods. This information is provided to the crew as well as to the resilient vehicle systems. Guidance on upset recovery, recovery time, and recovery state are also provided.

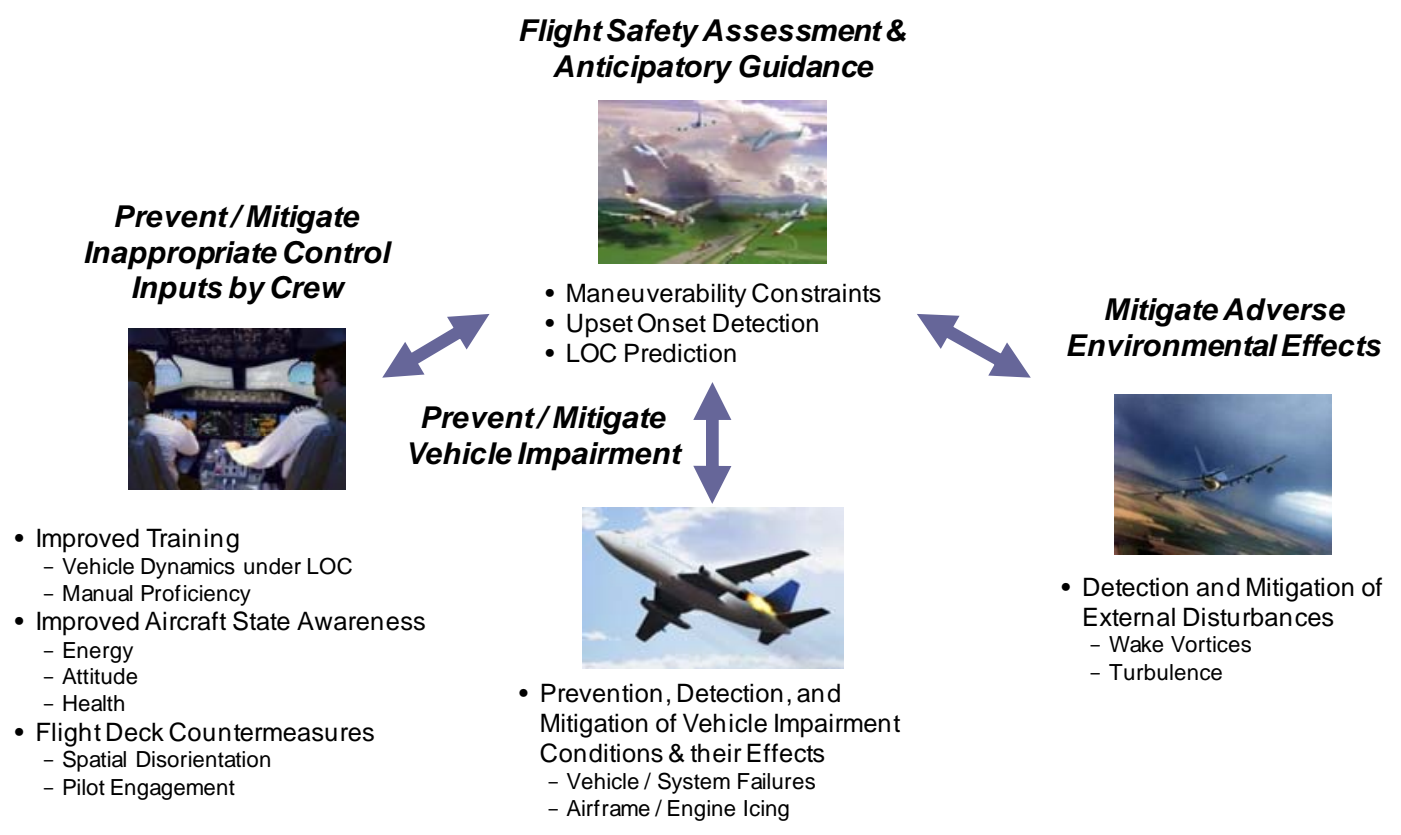

Figure 7. VSST integrated technologies for LOC prevention and recovery.

\section{E. Integrated GCS Research Description and System Architecture}

An overview of the GCS research component of Fig. 7 and the approach of Ref. 5 is depicted in Fig. 8 . Research and technology development is focused on onboard systems technologies for LOC prevention, with an emphasis on improved guidance and control under multiple hazards. In order to address improved SA under LOC hazards, novel methods for LOC prediction are being developed, as well as methods for dynamic envelope protection that enable protection in the presence of vehicle impairment conditions. Explicit methods for upset onset detection, prevention, and recovery will also be developed, as well as advanced control methods for the mitigation of multiple hazards and their impacts on vehicle dynamics and control. Partnerships will also be established through a LOC Working Group to assist in identifying emergent risks, defining LOC test scenarios for technology evaluation, developing validation requirements, and facilitating technology transfer.

In order to implement the research approach of Fig. 8, an integrated GCS architecture is needed as a research framework and for system development and integration. Figure 9 provides a preliminary integrated GCS architecture for LOC prevention and recovery. The colors depicted in Fig. 9 are consistent with those of the AIRSAFE System concept of Fig. 2; i.e., in Fig. 9: vehicle health state detection capabilities are indicated by green, vehicle flight safety state assessment and resilient guidance and control capabilities are shown in blue, crew-system interface information and support capabilities are shown in yellow, and onboard modeling capabilities are shaded purple. The signals depicted in Fig. 9 represent vector quantities and are defined as follows: " $x$ " is the vehicle state, "y" represents measurable outputs, "z" represents controlled variables (which can be mode-dependent), "u" represents control inputs (with subscript "p" denoting pilot input commands, and subscript "c" denoting control system commands), " $\mathrm{n}$ " represents noise signals, " $\mathrm{f}$ " represents failures (and in the case of jammed actuators, for example, can represent persistent asymmetric forces acting on the aircraft), and "d" represents external disturbances. 


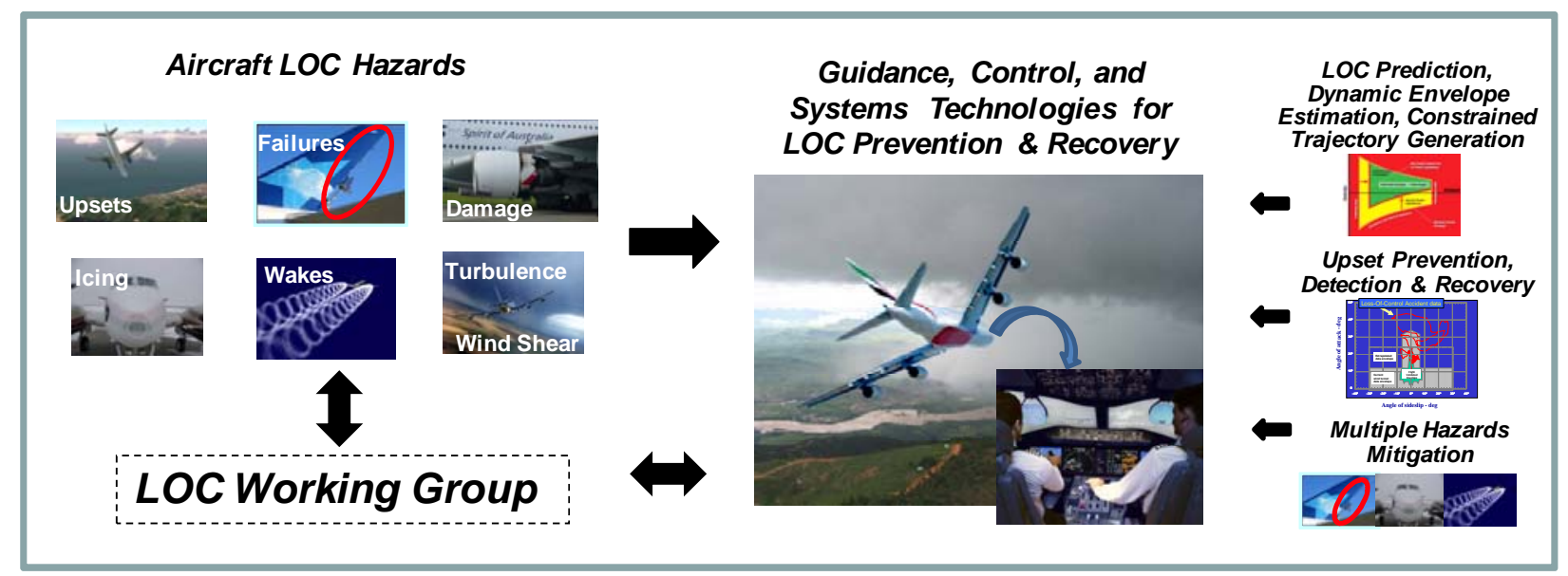

Figure 8. GCS research approach for LOC prevention and recovery.

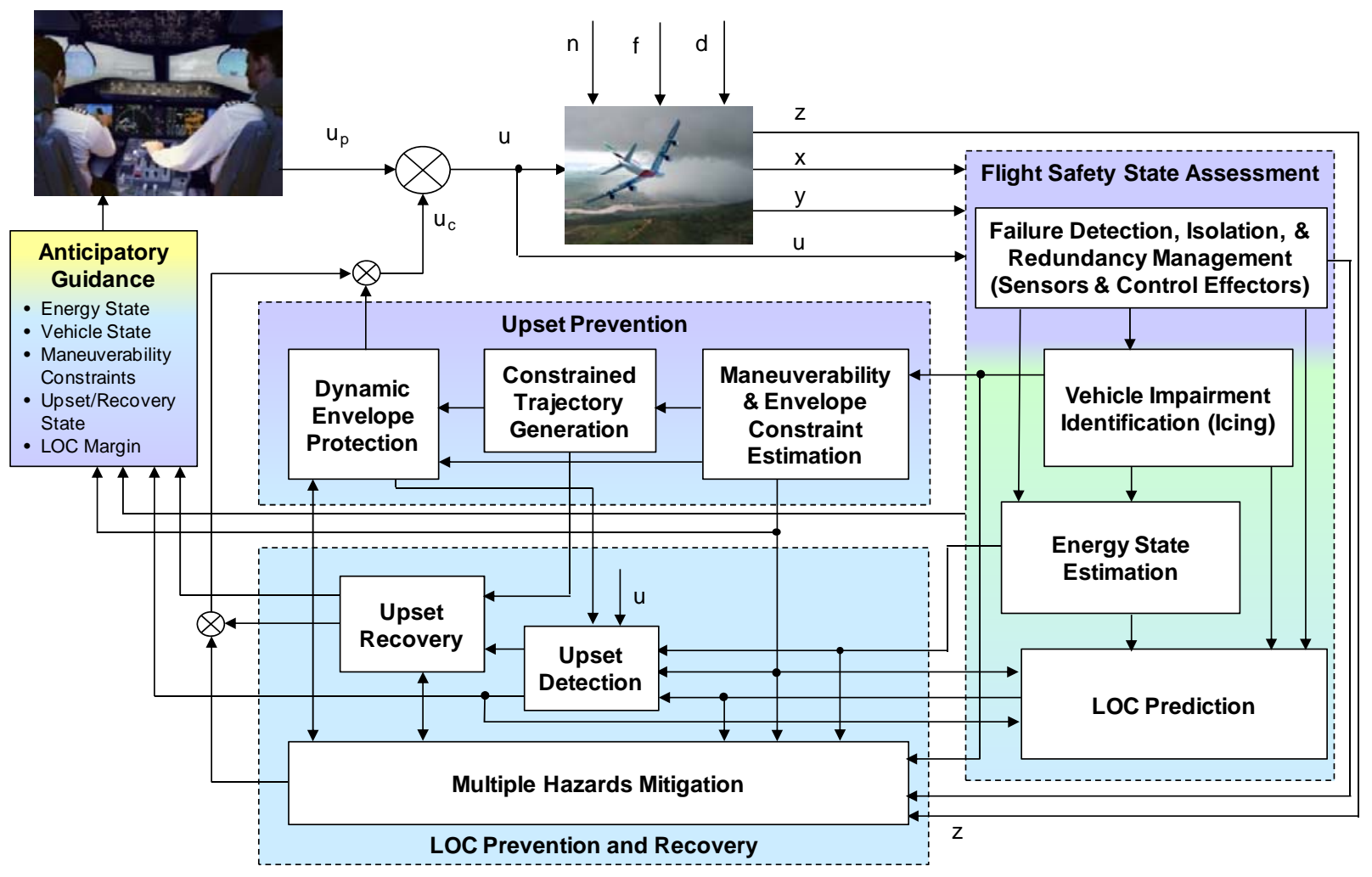

Figure 9. GCS architecture for LOC prevention and recovery.

Real-time assessment of flight safety is a key capability in providing improved onboard SA and control of aircraft operating under hazardous conditions. Assessment of flight safety (from a dynamics and control perspective) is inherently challenging because of the lack of a clear definition and the inherent nonlinear nature of events that lead to unsafe flight conditions. Moreover, flight safety assessment must be predictive to be the most beneficial. Near-term research into flight safety assessment will focus on failure detection, isolation, and mitigation (FDIM), vehicle impairment identification, energy state estimation, and LOC prediction. Control component failures are the focus of FDIM, with an emphasis on ensuring the integrity of the key sensors required for the flight 
safety assessment, upset prevention, and LOC prevention components. Energy state monitoring is based on current and predicted energy state and rate of change. This information is needed to prevent upsets resulting from loss of energy state awareness (LESA), particularly low-speed stalls. LOC prediction is based on the metrics defined in Ref. 9 or alternate metrics, and is included to provide advance warning of impending LOC so that unrecoverable conditions can be prevented. LOC is quantified in Ref. 9 by the exceedence of three or more of the five envelopes defined therein. Therefore, in the event of vehicle impairment, these envelopes would need to be updated by the maneuverability and envelope constraint estimation subsystem. As indicated in Fig. 9, much of this information would be provided to the crew for improved SA and anticipatory guidance. Research is currently being conducted in icing effects detection and identification in the presence of turbulence, ${ }^{14-15}$ engine icing effects modeling and detection, ${ }^{16}$ energy management, ${ }^{17}$ LOC prediction, ${ }^{18}$ and improved $\mathrm{SA}^{19-20}$.

Resilient control and anticipatory guidance methods are also of key importance in preventing LOC accidents, and are indicated by the middle two subsystems of Figure 9. These functions would supplement nominal aircraft systems, and would provide guidance information and control augmentations under LOC hazards when needed. Upset prevention technologies use information related to system failures or vehicle impairment conditions to dynamically determine changes to the safe operating envelopes of the impaired vehicle. This information is provided to the pilot and control system in the form of maneuverability and control constraints. The revised envelopes are also provided to the LOC prediction algorithms and for use in the generation of trajectories that can be safely flown by the impaired vehicle. These updated envelopes are also used by the resilient control system in providing a dynamic envelope protection capability for upset prevention. LOC prevention and recovery is accomplished through multiple hazards mitigation, early detection of vehicle upset, and upset recovery that ensures an unrecoverable state is not entered. Resilient control methods maintain stability and provide improved vehicle response and handling qualities under multiple hazards. These methods use all available control capability to overcome the effects of multiple hazards occurring individually or sequentially within a single LOC event.

Upset onset detection methods are being developed for identifying vehicle upset at an early stage in the presence of control input errors by the crew (e.g., those resulting from spatial disorientation or loss of energy state awareness) as well as other adverse conditions arising from vehicle impairment conditions or external hazards. The upset detection algorithms must be able to distinguish between the onset of a vehicle upset condition and normal or aggressive maneuvering without false alarms or missed detections. The detection of an upset triggers the generation of a recovery mechanism (based on safe trajectories for the current vehicle, either nominal or impaired), and recovery guidance cues are provided to the pilot. If safe recovery is not achieved or is not achievable by the pilot within a safe timeframe, an automatic recovery mechanism is engaged. The upset recovery methods provide the capabilities for early upset recovery as well as recovery from fully developed upsets for both nominal and impaired (but recoverable) aircraft.

The upset and recovery state of the vehicle is provided to the crew throughout for maintaining SA. Specialized flight deck technologies (being developed under CDM) are needed to provide this information to the crew in a clear and timely manner. Real-time modeling methods (e.g., system identification) are used in determining changes in vehicle dynamics resulting from icing, failures, or damage, as well as in determining changes to safe operating envelopes of the impaired vehicle. Research is currently being conducted in safe envelope estimation, ${ }^{21}$ trajectory generation under vehicle constraints, ${ }^{22}$ integration of dynamic envelope constraints into advance control methods, ${ }^{23-}$ ${ }^{24}$ integrated flight and propulsion control methods, ${ }^{25}$ robust aeroservoelastic control methods ${ }^{26}$ for (future) flexible aircraft, and upset recovery methods. ${ }^{27-28}$

\section{Technology Evaluation}

The VSST Project seeks to address cross-cutting aviation safety challenges that require integrated system effectiveness across technologies developed by the three technical challenges being addressed in VSST (see Fig. 3). Future vehicle-related safety considerations must mitigate emerging risks related to increasing automation and system complexity, increasing traffic density, new vehicle designs and materials, new operations, and greater fleet diversity. Technologies developed under VSST must enable the safe implementation of new capabilities (e.g., NextGen) and assure favorable outcomes under hazardous conditions (e.g., LOC precursors). While accomplishing this, it must be ensured that new vehicle safety technologies do no harm; i.e., that they themselves do not introduce new safety risks. The significant reduction of LOC as a key contributor to fatal aircraft accidents will require a coordinated effort among CDM, MVS, and ASC, and ultimately integrated system technologies that provide improved crew interfaces to support situation awareness and decision-making (CDM), real-time vehicle health management (MVS), and effective guidance and control under hazardous conditions (ASC). The safe realization of 
NextGen will also require vehicle capabilities that span each of the VSST TCs. The upper component of Fig. 3 represents the VSST subproject responsible for comprehensive testing and evaluation of the VSST technologies.

The validation of integrated technologies for LOC prevention and recovery, such as the proposed system of Figure 9, is a challenging problem. ${ }^{29}$ The objective of the evaluation process is to expose system weaknesses and vulnerabilities, and to identify safe and unsafe operational conditions, regions, and boundaries. The evaluation results should include a clear delineation of the coverage of LOC hazards provided by the proposed integrated system technologies. The following subsections provide an initial assessment of the potential effectiveness of the VSST technologies in terms of the approach of Fig. 6, and a brief discussion about technology validation. The preliminary assessment of potential technology effectiveness is similar to that presented in Ref. 5 for the AIRSAFE System concept. A full discussion about technology validation is provided in a companion paper (see Ref. 30).

\section{A. Potential Effectiveness in Preventing Aircraft LOC Accidents}

The technology development approach proposed in Ref. 5 was considered therein relative to its potential effectiveness in providing interventions at every stage of a generalized LOC sequence, and this approach appears to provide multiple intervention opportunities at all stages of the LOC sequence evaluated (see Fig. 6). A similar evaluation is presented in Figure 10 for the GCS architecture of Fig. 9 integrated with the VSST technologies of Fig. 7. The colored arrows and associated text describes interventions at each stage of the LOC sequence associated with each of the technology development areas depicted in Fig. 2 and 9. That is, purple correlates to vehicle dynamics modeling and simulation technologies, green reflects vehicle health management technologies, blue is indicative of flight safety management and resilient control technologies, and yellow represents crew interface technologies. The interventions will be discussed relative to each stage in the LOC sequence moving from left to right to illustrate potential technology inventions. One should note, however, that all functions (except upset recovery) are running continuously. Upset recovery is triggered when an upset is detected.

Starting at the left of Fig. 10, and before the flight even takes off, enhanced vehicle dynamics modeling methods provide insight into flight dynamics and control characteristics that occur under LOC hazards. The resulting improved database and models are used in developing LOC-enhanced simulations for improved crew training and resilient GCS system development and evaluation under realistic LOC conditions. Improved training methods provide a heightened ability to recognize vehicle upset conditions at an early stage and provide a higher level of manual handling proficiency. Improved design methods provide an inherent capability within the airframe and engine for failure prevention and containment. Once the flight takes off, flight safety state assessment technologies provide the capability to anticipate flight safety hazards or risks at initial inception or with some lead time, including sudden or significant changes to the vehicle energy state and LOC margin. The onboard modeling capability for characterizing hazards effects supports this assessment. Crew interface technologies provide the crew with notification of these flight safety impacts or risks. All of these technologies contribute to the avoidance of vehicle impairment and upset conditions, and, therefore, provide multiple opportunities for intervention very early in the LOC sequence.

Once a vehicle impairment or external hazard condition has occurred, the crew is better prepared to appropriately respond as a result of improved preflight training under realistic LOC conditions and improved real-time SA under the LOC conditions as they unfold. The onboard modeling technologies allow for models and databases to be rapidly updated to reflect the actual LOC condition being experienced and its impacts. This enables the accurate detection of impairment and hazard conditions by the VHM system, as well as associated diagnostics, prognostics, and containment functions. Flight safety assessment and resilient control technologies enable rapid assessment and prediction of off-nominal condition impacts and risks, mitigation of these effects through automatic control or guidance to the crew, and determination of achievable trajectories that the aircraft can safely fly. More specifically, flight safety assessment algorithms include LOC margin predictions, energy state impacts, and estimated vehicle impairment effects. Upset prevention algorithms provide estimates of revised flight envelopes that define safe regions of vehicle operation, which are used to provide dynamic envelope protection. LOC prevention algorithms provide control augmentation that ensures stability and provides improved handling qualities under multiple LOC hazards. Crew interface technologies enable the rapid and effective communication of the LOC conditions and their effects to the crew for improved SA, as well as guidance and cueing on control constraints and how to safely fly the aircraft under the current conditions. Providing appropriate information to the crew in order to formulate an optimum response is crucial in a LOC precursor condition due to the unforgiving flight environment and rapid onset of catastrophic conditions. These technologies working together provide the capability to rapidly detect and mitigate LOC precursor conditions while preventing an inappropriate response by the crew. 


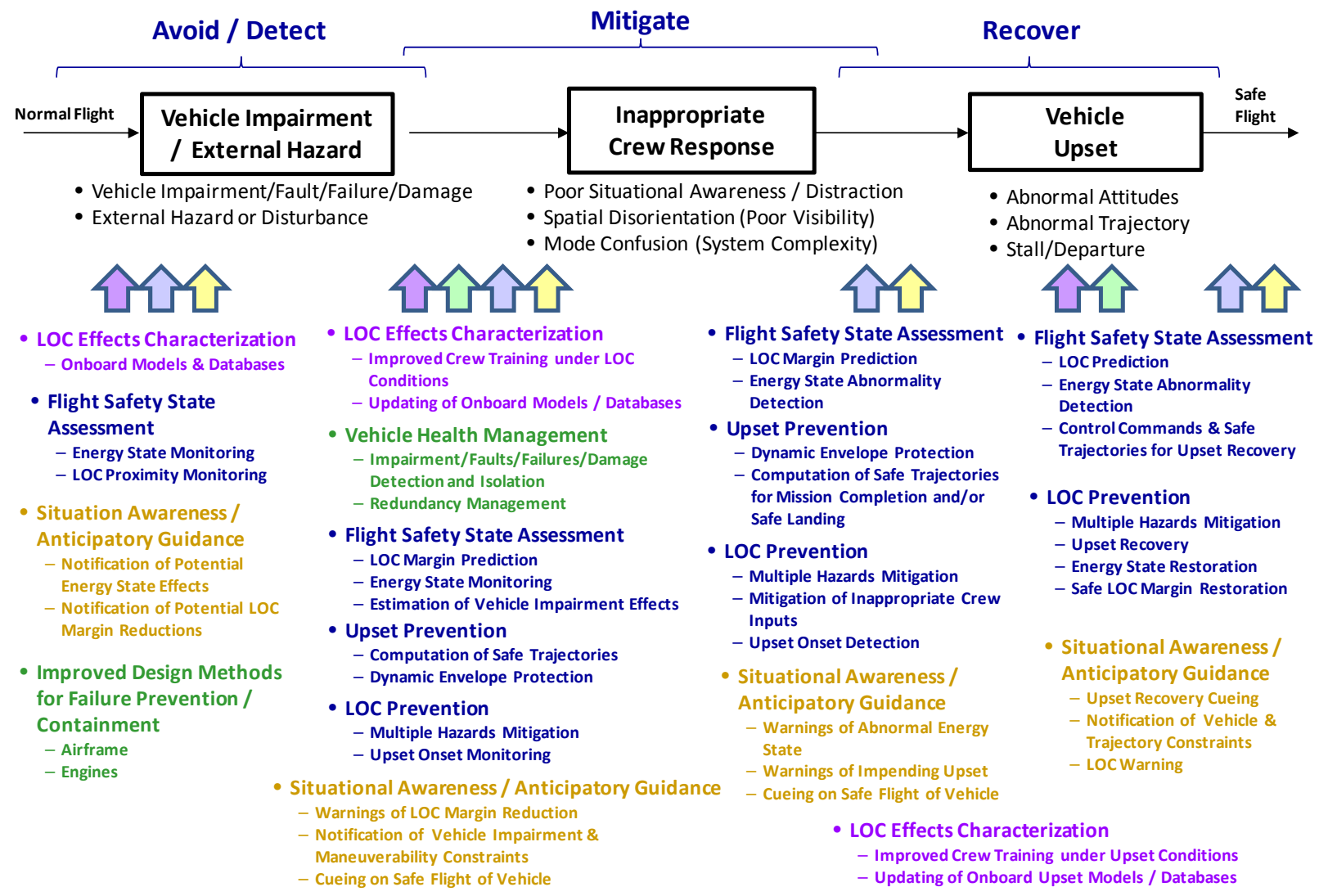

\section{Figure 10. Illustration of potential VSST technology effectiveness for LOC prevention and recovery based on an example LOC sequence.}

If an inappropriate crew response does occur, the flight safety management and resilient control technologies immediately detect LOC risk associated with the action (or inaction), and mitigate the effects to restore flight safety (e.g., to preserve LOC margin and a healthy energy state) while preventing a vehicle upset or damage. Dynamic envelope protection algorithms are working to prevent upsets, and upset detection algorithms are monitoring for the onset of a vehicle upset. Warnings are provided to the crew of impending flight safety risks associated with the inappropriate response (e.g., reduced LOC margin or energy state) and of mitigations being taken by the resilient system. Safe trajectories are generated for continuing the flight or landing the vehicle, and guidance for following them is provided to the crew. These technologies working together provide the capability to reduce the impact of inappropriate crew responses while mitigating the existing LOC precursor conditions and preventing a vehicle upset (or damage) condition.

If a vehicle upset condition does occur, the flight safety management and resilient control technologies provide the capability to detect and arrest the upset early in its progression as well as the capability to effect a full recovery, while continuing to mitigate the existing LOC precursor conditions and prevent entry into an unrecoverable condition. Safe recovery trajectories are generated for accomplishing the recovery in the context of the other LOC precursor conditions being experienced so as to prevent vehicle damage during the recovery. Throughout the upset detection and recovery, energy state abnormalities are detected and a healthy energy state maintained or restored and a safe LOC margin restored. Throughout the upset event, key information is communicated to the crew for improved SA and effective/optimal involvement by the crew. Moreover, throughout the upset event the crew is better able to recognize the upset condition and understand its effect on vehicle dynamics and control because of improved crew training in upset conditions enabled by enhanced modeling and simulation of realistic LOC conditions. The crew is also better able to manually fly the aircraft because of improved training to retain these skills. Finally, onboard models and databases are updated for future LOC events. All of these technologies working 
together provide the capability to mitigate the upset condition early in its inception and to fully recover from it while preventing a LOC accident from being the ultimate result.

\section{B. Technology Validation}

The integrated technologies depicted in Fig. 7 and 9 must be comprehensively evaluated using advanced analysis, simulation, and experimental test capabilities (see Ref. 29) to determine their effectiveness for LOC prevention and recovery. This is the subject of a companion paper. ${ }^{30}$ Validation of the individual and the integrated technologies is essential in ensuring that they are effective in assuring safe vehicle operations under current and future LOC risks (see Fig. 5), and that they themselves do no harm. For example, error propagation between subsystems (e.g., missed detections, false alarms, incorrect identifications, and incorrect decisions) must be thoroughly assessed for impacts to integrated system performance. As indicated in Ref. 29, this requires a validation capability that enables comprehensive technology evaluation under varying levels of integration. It also requires evaluation under realistic LOC scenarios that enable an evaluation of the level of coverage related to current and future LOC risks. Reference 30 provides a preliminary set of LOC test scenarios for use in evaluating the GCS and integrated VSST technologies being developed for LOC prevention and recovery, as well as a summary of the current VSST research on technology validation for LOC applications.

\section{Summary and Concluding Remarks}

Aircraft loss of control is one of the largest contributors to aircraft fatal accidents worldwide, and is a subject of research being conducted within NASA's Aviation Safety Program. This paper summarizes the near-term research approach being taken by NASA within the VSST Project for addressing LOC, with an emphasis on guidance, control, and systems technologies for LOC prevention and recovery. Hazards to be addressed over the next five years were determined based on an analysis of the most recent 10-year period from the accident set of Ref. 4 and a preliminary set of potential future hazards. It was determined that VSST research will focus on multiple hazards stemming from vehicle problems, external hazards, and crew errors. Vehicle-related hazards include system faults and failures and vehicle impairment conditions (airframe and engine) resulting from icing effects. External hazards include icing conditions and wake vortices, with wakes being considered as a potential future hazard resulting from high-density terminal area operations. Crew-related hazards include loss of energy state awareness and spatial disorientation, both of which can lead to various upsets including stalls and departures. The VSST research approach for addressing these hazards was presented, and a preliminary GCS architecture was defined. This architecture includes subsystems for flight safety assessment, upset prevention, and LOC prevention and recovery. Flight safety assessment will focus on failure detection, isolation, and mitigation, with an emphasis on sensors and control effectors, vehicle impairment identification for icing, energy state estimation, and LOC prediction. Upset prevention will focus on maneuverability and envelope constraint estimation, constrained trajectory generation, and dynamic envelope protection. LOC prevention and recovery will consist of resilient control methods for multiple hazards mitigation, upset onset detection, and upset recovery. Information from all of these subsystems will be provide to the crew. The potential effectiveness of this research was illustrated for an example LOC sequence, and a brief discussion of technology validation was presented. A full discussion of the validation of these technologies is presented in a companion paper (Ref. 30), as well as a preliminary set of LOC test scenarios for use in their evaluation. The LOC test scenarios are representative of the accident set analyzed in Ref. 4 as well as a set of future potential LOC risks, and includes a clear indication of their coverage. A LOC Working Group is being established to assist in identifying emergent risks, defining a complete set of LOC test scenarios, developing validation requirements, and facilitating technology transfer.

\section{Acknowledgments}

The research presented in this paper is dedicated to the memory and research contributions of Dr. Celeste M. Belcastro of NASA Langley Research Center, who was the beloved identical twin and research partner of the author. 


\section{References}

1 “Statistical Summary of Commercial Jet Airplane Accidents, Worldwide Operations, 1959-2010,” Boeing Commercial Airplanes, June 2011. URL: http://www.boeing.com/news/techissues/pdf/statsum.pdf.

${ }^{2}$ Lambregts, A. A., Nesemeier, G., Wilborn, J. E., and Newman, R. L., “Airplane Upsets: Old Problem, New Issues,” AIAA Modeling and Simulation Technologies Conference and Exhibit, 2008, AIAA 2008-6867.

${ }^{3}$ Evans, Joni K., “An Examination of In Flight Loss of Control Events During 1988-2004,” Alliant Techsystems, Inc., NASA Langley Research Center, Contract No.: TEAMS:NNL07AM99T/R1C0, Task No. 5.2, 2007.

${ }^{4}$ Belcastro, Christine M. and Foster, John V.: Aircraft Loss-of-Control Accident Analysis; AIAA Guidance, Navigation and Control Conference, Toronto, 2-5 August 2010.

${ }^{5}$ Belcastro, Christine M. and Jacobson, Steven, "Future Integrated Systems Concept for Preventing Aircraft Loss-of-Control Accidents," AIAA Guidance, Navigation and Control Conference, Toronto, 2-5 August 2010.

${ }^{6}$ Belcastro, Christine M., “Aircraft Loss of Control: Analysis and Requirements for Future Safety-Critical Systems and their Validation,” IEEE Asian Control Conference, Kaohsiung, Taiwan, 15-18 May 2011.

7 “Global Fatal Accident Review 1997-2006”, Civil Aviation Authority, Safety Regulation Group, CAP 776, 21 July 2008.

8 Joint Planning and Development Office, “Concept of Operations for the Next Generation Air Transportation System,” Version 3, October 2009, URL: http://www.jpdo.gov/library.asp [cited October 2009].

9 Joint Planning and Development Office, “National Aviation Safety Strategic Plan,” Version 1.0B, June 2011, URL: http://www.jpdo.gov/library/20110610_NASSP_V1.0b.pdf .

${ }^{10}$ Wilborn, J. E. and Foster, J. V., “Defining Commercial Aircraft Loss-of-Control: a Quantitative Approach,” AIAA Atmospheric Flight mechanics Conference and Exhibit, AIAA, Providence, Rhode Island, 16-19 August 2004.

${ }^{11}$ Casner, Stephen M., Geven, Richard W., and Williams, Kent T., "The Recognition of Abnormal Events Among Air Carrier Pilots,” Human Factors Journal, 2012 (Submitted).

12 Goupil, Philippe, “AIRBUS State of the Art and Practices on FDI and FTC in Flight Control System,” Control Engineering Practice, Vol. 19, pp. 524-539, April 2011.

${ }^{13}$ CAST, Safety Enhancement 30 Revision 5, "Mode Awareness and Energy State Management Aspects of Flight Deck Automation,” Final Report, August 2008, URL: http://www.cast-safety.org/pdf/cast_automation_aug08.pdf .

${ }^{14}$ Martos, Borja and Morelli, E.A., "Using Indirect Turbulence Measurements for Real-Time Parameter Estimation in Turbulent Air,” AIAA Guidance, Navigation, and Control Conference, 2012, Minneapolis, Minnesota.

${ }^{15}$ Morelli, Eugene A. and Cunningham, Kevin, “Aircraft Dynamic Modeling in Turbulence,” AIAA Guidance, Navigation, and Control Conference, 2012, Minneapolis, Minnesota.

${ }^{16}$ May, Ryan D., Simon, Donald L., and Guo, Ten-Huei, “Modeling and Detection of Ice Particle Accretion in Aircraft Engine Compression Systems,” AIAA Guidance, Navigation, and Control Conference, 2012, Minneapolis, Minnesota.

${ }^{17}$ Ganghi, Neha, Richards, Nathan D., Bateman, Alec J., Bolstad, Cheryl, and Costello, Anthony, "Pilot-in-the-Loop Demonstration of an Energy Monitor and Crew Alerting System,” AIAA Guidance, Navigation, and Control Conference, 2012, Minneapolis, Minnesota.

${ }^{18}$ Krishnakumar, Kalmanje, Stepanyan, Vahram, Barlow, Jonathan, and Govindarajan, Nithin, "Piloting on the Edge: Approaches to Real-Time Margin Estimation and Flight Control,” AIAA Guidance, Navigation, and Control Conference, 2012, Minneapolis, Minnesota.

${ }^{19}$ Conner, Kevin, Feyereisen, Thea, Morgan, John, and Bateman, Don, “Cockpit Displays and Annunciation to Help Reduce the Loss of Control (LOC) or Lack of Control (LAC) Accident Risks,” AIAA Guidance, Navigation, and Control Conference, 2012, Minneapolis, Minnesota. 
${ }^{20}$ Klyde, David H., Richards, Nathan, and Cogan, Bruce, "Use of Active Inceptor Cueing to Mitigate Pilot-Vehicle Loss of Control,” AIAA Guidance, Navigation, and Control Conference, 2012, Minneapolis, Minnesota.

${ }^{21}$ Allen, Robert C., Kwatny, Harry, and Bajpai, Gaurav, "Safe Set Protection and Restoration for Unimpaired and Impaired Aircraft,” AIAA Guidance, Navigation, and Control Conference, 2012, Minneapolis, Minnesota.

${ }^{22}$ Kaneshige, John, Panda, Ramesh, Hardy, Gordon, Steglinski, Mieczyslaw, Sharma, Shivanjli, and Benavides, Jose, “Constrained Trajectory Generation and Anticipatory Guidance for Loss of Control Prevention”. (In preparation)

${ }^{23}$ Wang, Xiaofeng, Xargay, Enric, Hovakymian, Naira, and Gregory, Irene, “Integration of Dynamic Flight Envelope into the $\mathrm{L}_{1}$ Adaptive Control Framework,” AIAA Guidance, Navigation, and Control Conference, 2012, Minneapolis, Minnesota.

${ }^{24}$ Wang, Chuan, Gregory, Irene M., and Cao, Chengyu, " $\mathrm{L}_{1}$ Adaptive Control with Output Constraints Applied to Flight Envelope Limiting,” AIAA Guidance, Navigation, and Control Conference, 2012, Minneapolis, Minnesota.

${ }^{25}$ Fuller, James, “Integrated Flight and Propulsion Control,” AIAA Guidance, Navigation, and Control Conference, 2012, Minneapolis, Minnesota.

${ }^{26}$ Moreno, Claudia, Balas, Gary J., and Seiler, Pete, "Robust Aeroservoelastic Control Using Linear, Parameter-Varying Techniques,” AIAA Guidance, Navigation, and Control Conference, 2012, Minneapolis, Minnesota.

${ }^{27}$ Ganghi, Neha, Richards, Nathan D., and Bateman, Alec J., “Desktop Simulator Demonstration of a Joint Human/Automated Upset Recovery System,” AIAA Guidance, Navigation, and Control Conference, 2012, Minneapolis, Minnesota.

${ }^{28}$ Richards, Nathan D., Ganghi, Neha, and Bateman, Alec J., "Improved Upset Recovery Strategies through Explicit Consideration of Pilot Dynamic Behavior,” AIAA Guidance, Navigation, and Control Conference, 2012, Minneapolis, Minnesota.

29 Belcastro, Christine M., "Validation and Verification of Future Integrated Safety-Critical Systems Operating under OffNominal Conditions,” AIAA Guidance, Navigation, and Control Conference, 2010, Toronto, Canada.

${ }^{30}$ Belcastro, Christine M., “Validation of Safety-Critical Systems for Aircraft Loss-of-Control Prevention and Recovery,” AIAA Guidance, Navigation, and Control Conference, 2012, Minneapolis, Minnesota. 